\title{
Little Bear's pulsating stars: Variable star census of UMi dSph Galaxy
}

\author{
K. Kinemuchi ${ }^{1,2, \star}$, E. Jeffery ${ }^{3, \star \star}$, C. Kuehn ${ }^{4, \star \star \star}, K$. Grabowski ${ }^{1,2, \star \star \star \star}$, and J. Nemec ${ }^{5, \dagger}$ \\ ${ }^{1}$ Apache Point Observatory, P.O. Box 59, Sunspot, NM 88349, USA \\ ${ }^{2}$ New Mexico State University, Department of Astronomy, Las Cruces, NM 88001, USA \\ ${ }^{3}$ Brigham Young University, Provo, UT, USA \\ ${ }^{4}$ University of Northern Colorado, Greeley, CO, USA \\ ${ }^{5}$ Camosun College, Victoria, B.C., Canada
}

\begin{abstract}
Recent observations and a photometric search for variable stars in the Ursa Minor dwarf spheroidal galaxy (UMi dSph) are presented. Our observations were taken at Apache Point Observatory in 2014 and 2016 using the 0.5m ARCSAT telescope and the West Mountain Observatory (WMO) 0.9m telescope of Brigham Young University in 2016. Previously known RR Lyrae stars in our field of view of the UMi dSph are identified, and we also catalog new variable star candidates. Tentative classifications are given for some of the new variable stars. We have conducted period searches with the data collected with the WMO telescope. Our ultimate goal is to create an updated catalog of variable stars in the UMi dSph and to compare the RR Lyrae stellar characteristics to other RR Lyrae stars found in the Local Group dSph galaxies.
\end{abstract}

\section{Introduction}

The Ursa Minor dwarf spheroidal galaxy (UMi dSph) is a Local Group member with a rich variable star population. RR Lyrae (RRL), anomalous Cepheids (AC), and eclipsing binary stars have been found and identified ([1, 4, 7]). Two different epochs of star formation are indicated via the RRL (older population) and the ACs (intermediate age). The UMi dSph is a metal-poor galaxy, and from the analysis of the RRL, it has been classified as an Oosterhoff-II (OoII) type object. UMi is one of a handful of known dwarf galaxies with a clear Oosterhoff classification.

The goals of this project are to revisit the UMi dSph galaxy and reinvestigate the variable star population. We present our variable star census based on the northeast portion of the galaxy. With our photometric data, we wil reevaluate and recalculate many of the stellar characteristics, in particular the pulsational characteristics.

\footnotetext{
${ }^{\star}$ kinemuchi@apo.nmsu.edu

$\star \star$ ejeffery@byu.edu

$\star \star \star$ charles. kuehn@unco.edu

$\star \star \star \star$ kgrabowski@apo.nmsu.edu

$\dagger$ jmn@isr.bc.ca
} 


\section{Data acquisition and reduction}

Data sets were collected at the $0.5 \mathrm{~m}$ Astrophysical Research Consortium Small Aperture Telescope (ARCSAT) at Apache Point Observatory and at the $0.9 \mathrm{~m}$ telescope at the West Mountain Observatory (WMO) of Brigham Young University. Due to the field coverage of the telescopes, we focused our survey on the northeast portion of the galaxy, centered at $\alpha=15: 09: 11.34$ and $\delta=67: 15: 51.7$. With the ARCSAT telescope, we obtained 100 epochs, and at WMO, over 200 epochs were observed.

Standard data reduction techniques were implemented, and to standardize the magnitudes, Landolt standards ([2]) were observed. Photometry was performed using DAOPHOT/ALLSTAR packages ([6]), and period analysis was done primarily with the dataset collected at WMO. Period solutions were obtained using the Supersmoother program ([5]), which uses a running linear regression algorithm. For amplitude, we used a basic measurement of the difference between maximum and minimum, omitting outliers $2.5 \sigma$ from the mean.

Table 1. Identified variable stars in the north portion of UMi dSph.

\begin{tabular}{cccccc}
\hline Star & $\alpha_{2000}(\mathrm{deg})$ & $\delta_{2000}(\mathrm{deg})$ & $V$ & Period $(\mathrm{d})$ & type \\
\hline V5 & 227.48149 & 67.474992 & 19.69 & 0.766705 & $\mathrm{ab}$ \\
V6 & 227.49143 & 67.464966 & 18.17 & 0.725528 & $\mathrm{AC}$ \\
V7 & 227.44961 & 67.454614 & 19.61 & 0.690328 & $\mathrm{ab}$ \\
V8 & 227.294 & 67.451651 & 19.70 & 0.553879 & $\mathrm{ab}$ \\
V9 & 227.53442 & 67.419453 & 19.59 & 0.356460 & $\mathrm{c} ?$ \\
V10 & 227.58564 & 67.405209 & 19.69 & 0.617496 & $\mathrm{ab}$ \\
V11 & 227.63938 & 67.417687 & 18.88 & 0.673431 & $\mathrm{AC}$ \\
V12 & 227.63475 & 67.395875 & 19.65 & 0.771735 & $\mathrm{ab}$ \\
V13 & 227.46745 & 67.403259 & 19.66 & 0.646050 & $\mathrm{ab}$ \\
V19 & 227.53903 & 67.357643 & 19.67 & 0.341565 & $\mathrm{c}$ \\
V24 & 227.54186 & 67.310734 & 19.83 & 0.301081 & $\mathrm{c} ?$ \\
V28 & 227.6731 & 67.278736 & 19.70 & 0.308163 & $\mathrm{c} ?$ \\
V29 & 227.62463 & 67.252691 & 19.73 & 0.409747 & $\mathrm{c} ?$ \\
V41 & 227.46443 & 67.232054 & 19.84 & 0.490488 & $\mathrm{ab}$ \\
V42 & 227.59471 & 67.209778 & 19.74 & 0.646473 & $\mathrm{ab}$ \\
V45 & 227.35121 & 67.207436 & 19.02 & 0.216662 & $?$ \\
V48 & 227.18208 & 67.218741 & 19.69 & 0.687258 & $\mathrm{ab}$ \\
V49 & 227.18687 & 67.212797 & 19.73 & 0.415464 & $\mathrm{c} ?$ \\
V78 & 227.29384 & 67.38137 & 19.69 & 0.372640 & $\mathrm{c} ?$ \\
V79 & 227.38367 & 67.328828 & 19.70 & 0.313023 & $\mathrm{c} ?$ \\
V80 & 227.41875 & 67.3245 & 18.74 & 0.512738 & $?$ \\
v1540* & 227.88001 & 67.426652 & 19.79 & 0.714490 & $\mathrm{ab}$ \\
v1678* & 227.19679 & 67.413053 & 19.75 & 0.336900 & $\mathrm{c} ?$ \\
v1895* & 227.09781 & 67.386081 & 19.45 & 0.490887 & $\mathrm{ab}$ \\
v2078* & 227.83088 & 67.361948 & 19.73 & 0.719031 & $\mathrm{ab}$ \\
v3176* & 227.32619 & 67.236128 & 19.81 & 0.327060 & $\mathrm{c} ?$ \\
\hline & & $*$ & Newly identified star. & &
\end{tabular}




\section{RR Lyrae results}

From the 543 objects identified and photometered, we find 22 RR Lyrae candidates. Cross-matching with the finding charts and variable star catalog of [4], we find 12 ab-type and 10 c-type RRL stars. We additionally find 2 ACs, V6 and V11 (using Nemec's IDs). In Table 1, we provide our identified variable stars' IDs, equatorial coordinates (as determined from astrometry. net, [3]), mean $V$ magnitude, period solution, and variable star type. The star ID is the same as in [4], unless it is denoted with an asterisk, then it is a newly identified star.

\section{Curious case of V80}

The variable star identified as V80 in $[1,4,7]$ has an unclear classification. This star's variability appears real in our datasets from 2016. We find the amplitude of this star to be closer to the published value in [4], but the periodicity is longer than in any of the published literature. The period solution for V80 was 0.512738 days. We cannot conclusively classify this variable star at this time. Applying frequency analysis will help understand the nature.

\section{Future Work}

Additional data are expected to be collected in March 2017 and will cover the southern regions of UMi dSph that has been missed. With the fairly complete coverage of the pulsation cycle to many of the RRab stars, we will calculate the $[\mathrm{Fe} / \mathrm{H}]$ metallicities through Fourier decomposition parameters. This work will allow us to obtain an independent measurement of the dSph galaxy's metallicity.

Acknowledgments: K.K. would like to thank Suzanne Hawley, director of ARCSAT for the generous time granted for this project. Professional development funds through the Astrophysical Research Consortium allowed K.K. to present this work at this conference and is gratefully acknowledged.

\section{References}

[1] Kholopov, P. N., Peremennye Zvezdy, 18, 117 (1971)

[2] Landolt, A. U., AJ, 104, 340 (1992)

[3] Lang, D., Hogg, D. W., Mierle, K., Blanton, M., \& Roweis, S., AJ, 139, 1782 (2010)

[4] Nemec, J. M., Wehlau, A., \& Mendes de Oliveira, C., AJ, 96, 528 (1988)

[5] Reimann, J. D., Ph.D. Thesis (1994)

[6] Stetson, P. B., PASP, 99, 191 (1987)

[7] van Agt, S. L. T. J., Bulletin of the Astronomical Institutes of the Netherlands, 19, 275 (1967) 